\title{
Young Galaxy Candidates in the Hubble Frontier Fields. IV. MACS J1149.5+2223
}

\author{
Wei Zheng ${ }^{1}$, Adi Zitrin ${ }^{2,3,16}$, Leopoldo Infante ${ }^{4}$, Nicolas Laporte ${ }^{5}$, Xingxing Huang ${ }^{6}$, John Moustakas ${ }^{7}$, Holland C. Ford ${ }^{1}$, \\ Xinwen Shu ${ }^{6,8}$, Junxian Wang ${ }^{6}$, Jose M. Diego ${ }^{9}$, Franz E. Bauer ${ }^{4,10,11}$, Paulina Troncoso Iribarren ${ }^{4}$, Tom Broadhurst ${ }^{12,13}$, and \\ Alberto Molino ${ }^{14,15}$ \\ ${ }^{1}$ Department of Physics and Astronomy, Johns Hopkins University, Baltimore, MD 21218, USA \\ ${ }^{2}$ Cahill Center for Astronomy and Astrophysics, California Institute of Technology, MS 249-17, Pasadena, CA 91125, USA \\ ${ }^{3}$ Physics Department, Ben-Gurion University of the Negev, P.O. Box 653, Be'er-Sheva 84105, Israel \\ ${ }^{4}$ Instituto de Astrofísica and Centro de Astroingeniería, Facultad de Física, Pontificia Universidad Católica de Chile, Santiago 22, Chile \\ ${ }^{5}$ Department of Physics and Astronomy, University College London, London NW1 2PS, UK \\ ${ }^{6}$ Department of Astronomy, University of Science and Technology of China, Hefei, Anhui 230026, China \\ ${ }^{7}$ Department of Physics and Astronomy, Siena College, Loudonville, NY 12211, USA \\ ${ }^{8}$ Department of Physics, Anhui Normal University, Wuhu, Anhui, 241000, China \\ ${ }^{9}$ IFCA, Instituto de Física de Cantabria, UC-CSIC, s/n. E-39005 Santander, Spain \\ ${ }^{10}$ Millennium Institute of Astrophysics, Santiago 22, Chile \\ ${ }^{11}$ Space Science Institute, Boulder, CO 80301, USA \\ ${ }^{12}$ Department of Theoretical Physics, University of Basque Country UPV/EHU, Bilbao, Spain \\ ${ }_{13}$ IKERBASQUE, Basque Foundation for Science, Bilbao, Spain \\ ${ }^{14}$ Instituto de Astronomía, Geofísica e Ciências Atmosféricas, Universidade de São Paulo, Cidade Universitária, 05508-090, São Paulo, Brazil \\ ${ }^{15}$ Instituto de Astrofísica de Andalucía-CSIC, Glorieta de la Astronomía, s/n. E-18008, Granada, Spain \\ Received 2016 September 6; revised 2017 January 12; accepted 2017 January 29; published 2017 February 23
}

\begin{abstract}
We search for high-redshift dropout galaxies behind the Hubble Frontier Fields (HFF) galaxy cluster MACS $\mathrm{J} 1149.5+2223$, a powerful cosmic lens that has revealed a number of unique objects in its field. Using the deep images from the Hubble and Spitzer space telescopes, we find 11 galaxies at $z>7$ in the MACS J1149.5+2223 cluster field, and 11 in its parallel field. The high-redshift nature of the bright $z \simeq 9.6$ galaxy MACS1149-JD, previously reported by Zheng et al., is further supported by non-detection in the extremely deep optical images from the HFF campaign. With the new photometry, the best photometric redshift solution for MACS1149-JD reduces slightly to $z=9.44 \pm 0.12$. The young galaxy has an estimated stellar mass of $(7 \pm 2) \times 10^{8} M_{\odot}$, and was formed at $z=13.2_{-1.6}^{+1.9}$ when the universe was $\approx 300 \mathrm{Myr}$ old. Data available for the first four HFF clusters have already enabled us to find faint galaxies to an intrinsic magnitude of $M_{U V} \simeq-15.5$, approximately a factor of 10 deeper than the parallel fields.
\end{abstract}

Key words: cosmology: observations - galaxies: clusters: individual (MACS J1149.5+2223) - galaxies: highredshift - gravitational lensing: strong

\section{Introduction}

The galaxy cluster MACS J1149.5+2223 $(z=0.54$, Ebeling et al. 2007, MACS1149 hereafter) is one of the most interesting and best studied cosmic lenses. Its strong lensing power is demonstrated by many sets of multiply imaged galaxies, including the largest known lensed images of a face-on spiral galaxy at $z=1.491$, whose highly magnified images display little distortion (Zitrin \& Broadhurst 2009; Smith et al. 2009). The first multiply imaged supernova was discovered in the lensed images of this spiral, forming an Einstein cross (Kelly et al. 2015). The supernova reappeared a year later in another counter image of the spiral as predicted by lensing models (Kelly et al. 2016; Rodney et al. 2016; Treu et al. 2016).

Because of its high magnification power, MACS1149 was selected for the CLASH program (The Cluster Lensing And Supernova survey with Hubble, Postman et al. 2012), which obtained HST images of 25 galaxy clusters in 16 broad bands between $0.2-1.7 \mu \mathrm{m}$ to a depth of $\mathrm{AB}$ magnitude $\simeq 27$. A relatively bright, young galaxy, M1149-JD at $z \simeq 9.6$ and magnitude $\simeq 26$, was discovered approximately one arcminute north of the cluster center (Zheng et al. 2012b, Z12 hereafter). Lensing models based on early data (Zitrin \& Broadhurst 2009; Zitrin et al. 2011) suggested that the source was magnified by

\footnotetext{
${ }^{16}$ Hubble Fellow.
}

approximately a factor of 15 , and that no counter images were expected (although of note, the models lack sufficient constraints in this region).

MACS1149 is also one of the six targets of the Hubble Frontier Fields program (HFF; Lotz et al. 2016), whose chief goal is reaching the deepest universe with the aid of gravitational lensing. This is particularly important for galaxies at $z \gtrsim 7$ as they are believed to be the main ionization source of the intergalactic medium during this period (Loeb \& Barkana 2001; Bouwens et al. 2008, 2010, 2012; Atek et al. 2015; Robertson et al. 2015), but most of them are too faint to be detected in random deep fields. In Cycle 21, Abell 2744 and MACS J0416.1-2403 (MACS0416) were observed, and in Cycle 22 MACS J0717.5+3745 (MACS0717) and MACS1149 were observed. In Cycle 23, Abell S1063 and Abell 370 were observed. Thanks to the additional, intensive monitoring observations of the multiply imaged supernova (PIs: Kelly, Rodney), the HST/WFC3-IR data of MACS1149 are the deepest among all clusters.

The HFF clusters have therefore been the subject of many studies searching for high-redshift Lyman-break galaxies (LBG; Atek et al. 2014, 2015; Ishigaki et al. 2015; McLeod et al. 2015; Kawamata et al. 2016; Livermore et al. 2016). An important result is the faint-end luminosity function (LF) of galaxies down to an intrinsic absolute magnitude of 
Table 1

Summary of Observations

\begin{tabular}{|c|c|c|c|c|}
\hline Telescope & Band & $\begin{array}{l}\text { Date } \\
\text { CLASH-HFF }\end{array}$ & $\begin{array}{l}\text { Exposure Time } \\
(\mathrm{ks})\end{array}$ & $\begin{array}{c}\text { Limiting } \\
\text { Magnitude }(5 \sigma)^{\mathrm{a}}\end{array}$ \\
\hline$H S T$ & F160W & 2010 Dec-2016 Feb & 107.6 & 28.9 \\
\hline$H S T$ & F140W & 2011 Jan-2014 Nov & 30.6 & 28.6 \\
\hline$H S T$ & F125W & 2010 Dec-2016 Feb & 66.9 & 28.9 \\
\hline$H S T$ & F105W & 2011 Jan-2016 Feb & 85.5 & 29.1 \\
\hline$H S T$ & F814W & 2004 Apr-2015 May & 104.2 & 29.3 \\
\hline$H S T$ & F606W & 2011 Jan-2015 May & 26.9 & 29.0 \\
\hline$H S T$ & F435W & 2011 Feb-2015 May & 44.7 & 29.2 \\
\hline Spitzer & IRAC1 & 2010 Jul-2015 Mar & 357.7 & 25.5 \\
\hline Spitzer & IRAC2 & 2010 Jul-2015 Mar & 357.7 & 25.6 \\
\hline
\end{tabular}

Note.

a Near the cluster center, the detection limits are lower by $\approx 0.2-0.3$ mag, because of higher sky background levels from BCGs.

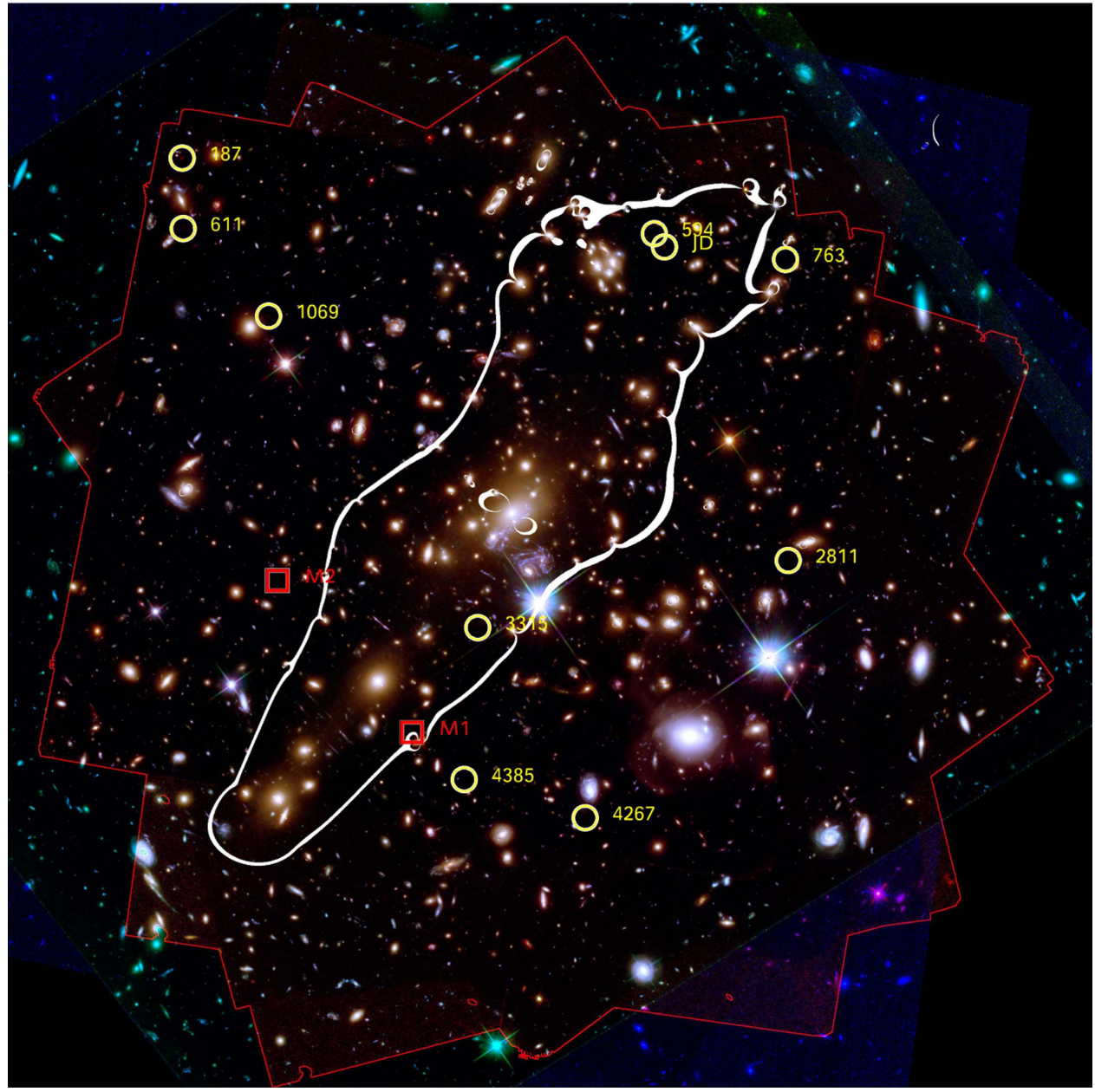

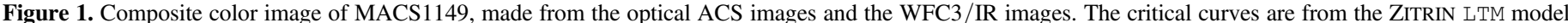

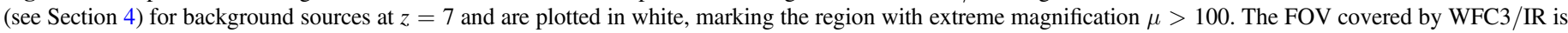
marked by a red polygon. Yellow circles mark the LBGs at $z>7$, and red boxes mark a possibly related $z \simeq 7.1$ system.

$M_{U V} \simeq-15.5$, as estimated around the rest-frame wavelength of $1500 \AA$. This is considerably deeper than the results from the Hubble Deep Field (Bouwens et al. 2015), manifesting the uniqueness of the HFF program.

Our group has been carrying out a systematic study of highredshift LBGs in the HFF clusters, reported in a series of papers to date (Zheng et al. 2014; Zitrin et al. 2014 on Abell 2744; Infante et al. 2015 on MACS0416; and Laporte et al. 2016 on
MACS0717). Here, we extend our search to MACS1149 and report 11 candidates at $z>7$ in the field of MACS1149 and 11 in its parallel field.

\section{Data}

The HFF observations of MACS1149 (GO/DD 13504, PI: Lotz) were carried out between 2013 November 2 and 2015 May 19. Additional archival images were obtained from three 


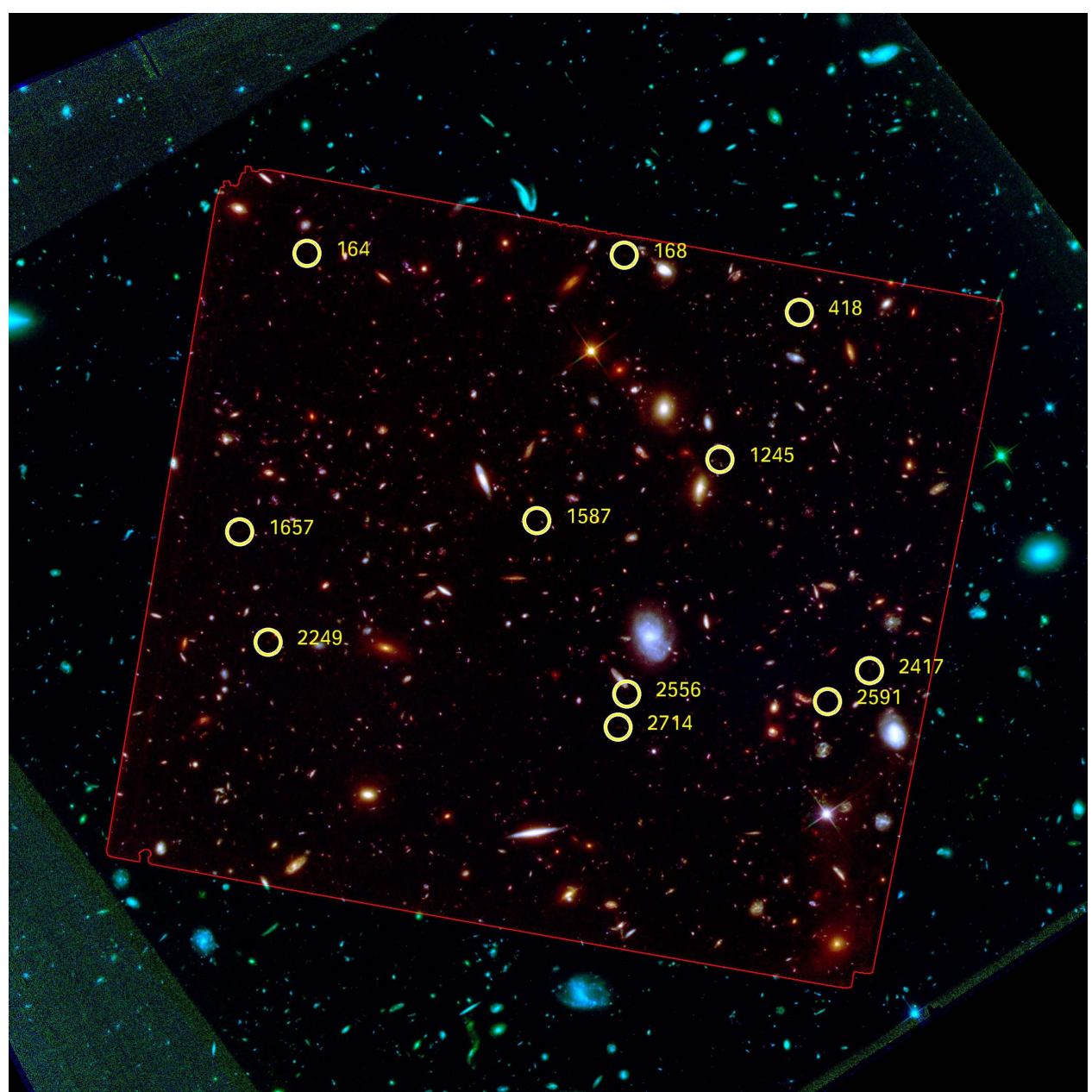

Figure 2. Composite color image of the MACS1149 parallel field, made from the optical ACS images and the WFC3/IR F140W image. The FOV of WFC3/IR is marked by a red box, and yellow symbols mark the LBGs at $z>7$.

groups of resource: (1) the early images (GO 9722, PI: Ebeling; GO 10493, PI: Gal-Yam); (2) the CLASH multi-band images (GO 12068, PI: Postman); and (3) recent WFC3/IR images (GO 14041, 14199, PI: Kelly; GO 13790, PI: Rodney; and GO 13459, PI: Treu). Table 1 lists the exposure times and limiting magnitudes for the imaging data used in our analysis.

We processed the HST data using APLUS (Zheng et al. 2012a). The calibrated images from the HST instrument pipelines, namely the $f c$ images for ACS (corrected for the detector charge transfer efficiency) and $f t$ images for WFC3/ IR, were retrieved. The pixel scale for the final mosaic images is 0 "'065. Figure 1 displays a composite color image of the cluster field, and Figure 2 the parallel field. The source catalogs are generated with SExtractor (Bertin \& Arnouts 1996) in dual mode, using the summed WFC3/IR image as a reference. As a comparison, we ran SExtractor with the public HFF images (Koekemoer et al. 2016) and checked with the measurements in Table 2. The results confirm that the photometry of our candidates agrees within the stated errors.

As part of the HFF campaign, deep Spitzer/IRAC images of MACS1149 were obtained between 2014 March and 2015 March in Channels 1 and 2, using Director's Discretionary Time (Program 90260, PI: Soifer). The effective exposure time in each channel, including that of the archival data (Program 60034, PI: Egami and 90009, PI: Bradač) obtained in 2010, 2011 and 2013, is $\simeq 358 \mathrm{ks}$. The IRAC corrected Basic
Calibrated Data (cBCD) images were processed with MOPEX (Makovoz \& Khan 2005) and sampled to a final pixel scale of 0 " 6 . The estimated $1 \sigma$ limiting magnitude is 27.3 for IRAC channel 1 (IRAC1, $3.6 \mu \mathrm{m}$ ) and 27.4 for channel 2 (IRAC2, $4.5 \mu \mathrm{m})$. More details of the processing of HST and IRAC data can be found in Zheng et al. (2014).

\section{Selection}

Our selection consists of two steps: a color selection and a photometric redshift selection. We first searched for LBGs using their distinct color around $0.1216(1+z) \mu \mathrm{m}$. At $z>7$, these sources are optical dropouts. At $z \simeq 7-8$, the Ly $\alpha$ break is at $\simeq 1 \mu \mathrm{m}$, between the F $814 \mathrm{~W}$ and F125W bands. Our selection criteria for $z \simeq 7-8$, in units of magnitude, are as follows:

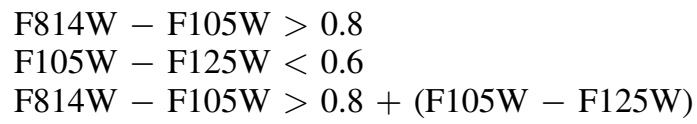

For $z \simeq 8-9$, the break is at $\simeq 1.15 \mu \mathrm{m}$, between the F105W and F140W bands:

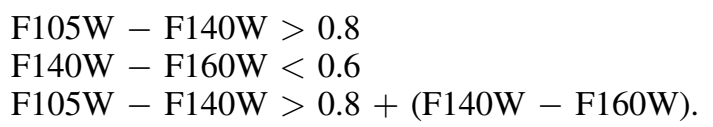


Table 2

Candidates at $z>7$ in MACS1149

\begin{tabular}{|c|c|c|c|c|c|c|c|c|c|c|c|c|}
\hline Name & $\begin{array}{l}\text { Photometric } \\
\text { Redshift }\end{array}$ & $\begin{array}{l}\text { R.A. } \\
\text { (J2000) }\end{array}$ & $\begin{array}{l}\text { Decl. } \\
\text { (J2000) }\end{array}$ & F160W & F140W & $\mathrm{F} 125 \mathrm{~W}$ & F105W & F814W & IRAC1 & IRAC2 & $\mu^{\mathrm{b}}$ & $\begin{array}{l}\text { Other } \\
\text { Reference }\end{array}$ \\
\hline 611 & $9.6 \pm 0.2$ & 177.41769 & 22.41369 & $26.73 \pm 0.04$ & $27.12 \pm 0.08$ & $27.75 \pm 0.15$ & $28.82 \pm 0.27$ & $>31.0$ & $\ldots$ & $\ldots$ & $1.5_{-0.4}^{+0.2}$ & K16 (Y2) \\
\hline JD (663) & $9.44_{-0.13}^{+0.11}$ & 177.38995 & 22.41271 & $25.70 \pm 0.01$ & $25.88 \pm 0.02$ & $26.57 \pm 0.03$ & $>30.7$ & $29.3 \pm 0.3$ & $25.64 \pm 0.17$ & $24.73 \pm 0.07$ & $11.5_{-3.1}^{+3.3}$ & Z12, K16 (YJ1) \\
\hline 594 & $9.1 \pm 0.5$ & 177.39055 & 22.41341 & $28.07 \pm 0.10$ & $28.09 \pm 0.13$ & $28.73 \pm 0.21$ & $>30.7$ & $>31.0$ & $\ldots$ & $\ldots$ & $10.4_{-2.2}^{+9.0}$ & K16 (YJ4) \\
\hline 3315 & $8.7_{-0.3}^{+0.4}$ & 177.40073 & 22.39244 & $28.11 \pm 0.08$ & $28.13 \pm 0.10$ & $28.34 \pm 0.11$ & $>30.7$ & $>31.0$ & $\ldots$ & $\ldots$ & $20_{-14}^{+22}$ & K16 (YJ3) \\
\hline 4267 & $8.3_{-0.7}^{+0.3}$ & 177.39453 & 22.38231 & $28.29 \pm 0.09$ & $28.04 \pm 0.09$ & $28.32 \pm 0.09$ & $29.49 \pm 0.27$ & $>31.0$ & $>26.1$ & $>25.5$ & $1.7_{-0.3}^{+0.2}$ & \\
\hline 2811 & $8.0_{-0.7}^{+0.4}$ & 177.38284 & 22.39600 & $27.75 \pm 0.09$ & $27.85 \pm 0.14$ & $27.70 \pm 0.11$ & $28.98 \pm 0.30$ & $>31.0$ & $\ldots$ & $\ldots$ & $2.4_{-0.5}^{+1.4}$ & \\
\hline 763 & $7.7_{-0.6}^{+0.3}$ & 177.38298 & 22.41203 & $28.05 \pm 0.08$ & $27.88 \pm 0.08$ & $28.03 \pm 0.09$ & $28.75 \pm 0.12$ & $>31.0$ & $>25.6$ & $>25.6$ & $3.6_{-1.3}^{+3.7}$ & K16 (i19) \\
\hline 4385 & $7.7_{-1.3}^{+0.8}$ & 177.40151 & 22.38434 & $28.62 \pm 0.09$ & $28.36 \pm 0.10$ & $28.72 \pm 0.10$ & $29.62 \pm 0.21$ & $>31.0$ & $\ldots$ & $\ldots$ & $2.6_{-0.1}^{+1.3}$ & \\
\hline 187 & $7.3 \pm 0.1$ & 177.41776 & 22.41744 & $24.88 \pm 0.02$ & $24.87 \pm 0.02$ & $24.92 \pm 0.02$ & $25.31 \pm 0.02$ & $28.3 \pm 0.3$ & $\cdots$ & $\ldots$ & $1.4_{-0.3}^{+0.2}$ & K16 (i1) \\
\hline 1069 & $7.3_{-0.7}^{+0.3}$ & 177.41278 & 22.40902 & $27.44 \pm 0.07$ & $27.21 \pm 0.07$ & $27.45 \pm 0.08$ & $27.77 \pm 0.09$ & $>31.0$ & $\cdots$ & $\ldots$ & $1.8_{-0.3}^{+0.2}$ & K16 (i11) \\
\hline M1- $4012^{\mathrm{c}}$ & $7.2_{-0.4}^{+0.3}$ & 177.40451 & 22.38688 & $27.01 \pm 0.06$ & $26.83 \pm 0.07$ & $27.08 \pm 0.07$ & $27.31 \pm 0.08$ & $>31.0$ & $>25.5^{\mathrm{d}}$ & $>24.9^{\mathrm{d}}$ & $7.0_{-2.4}^{+39}$ & K16 (39.2) \\
\hline M1-4023 & $7.2 \pm 0.3$ & 177.40460 & 22.38668 & $26.48 \pm 0.10$ & $26.55 \pm 0.10$ & $26.88 \pm 0.13$ & $26.93 \pm 0.11$ & $27.7 \pm 0.2$ & $>25.5^{\mathrm{d}}$ & $>24.9^{\mathrm{d}}$ & $7.0_{-24}^{+39}$ & K16 (21.1) \\
\hline M2-2952 & $6.9_{-1.5}^{+0.3}$ & 177.41226 & 22.39497 & $27.72 \pm 0.08$ & $27.67 \pm 0.10$ & $27.60 \pm 0.07$ & $27.72 \pm 0.07$ & $>31.0$ & $>25.5^{\mathrm{d}}$ & $>26.5^{\mathrm{d}}$ & $6.6_{-1.9}^{+3.1}$ & K16 (i24) \\
\hline M2-2963 & $7.1_{-1.1}^{+0.3}$ & 177.41220 & 22.39489 & $27.89 \pm 0.08$ & $27.69 \pm 0.09$ & $27.78 \pm 0.08$ & $27.97 \pm 0.08$ & $>31.0$ & $>25.5^{\mathrm{d}}$ & $>26.5^{\mathrm{d}}$ & $6.9_{-2.0}^{+3.4}$ & K16 (i26) \\
\hline
\end{tabular}

Notes.

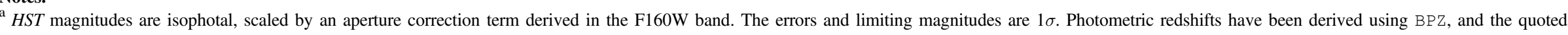
uncertainties indicate the $68 \%$ confidence interval.

Magnification factor is the mean of seven models and the range of five middle-ranked models after excluding the highest and lowest values.

${ }^{c} \mathrm{BPZ}$ is uncertain because of contamination from a nearby object.

${ }^{\mathrm{d}}$ Upper limit for both components, which are unresolved in IRAC images. 
For candidates at $z>9$ the break is between the F125W and F160W bands:

$$
\mathrm{F} 125 \mathrm{~W}-\mathrm{F} 160 \mathrm{~W}>0.8 \text {. }
$$

We require that a candidate is not detected above $1 \sigma$ in a summed image blueward of the selection bands defined above. For objects at $z \simeq 7$, this requires a non-detection in a summed image of the F606W and F435W bands, while for candidates at $z>8$ this requires a non-detection in the stacked optical detection image. For candidates at $z>9$, a non-detection is also required in the $\mathrm{F} 105 \mathrm{~W}$ or even the F125W band.

In addition to the color selection criteria described above, we excluded candidates lying within one arcsecond of the detector edges, in order to mitigate potentially spurious detections. Approximately 150 candidates were initially selected in the cluster field, and we visually inspected them. A large portion of these candidates were excluded as artifacts following a visual inspection, and often also due to an incomplete image coverage in certain bands. Some others are excluded as they are near stellar diffraction spikes, which are difficult to remove because the HFF WFC3/IR exposures were obtained at the same position angle. Through these steps the number of candidates was reduced to approximately 30 , enabling us to carry out IRAC photometry manually with reasonable effort. The candidates with a color decrement of F160W-IRAC1 $>3$ were rejected, as they are most likely extremely red objects at lower redshifts (Wang et al. 2012). A similar procedure was performed in the parallel field.

The IRAC images of our candidates suffer from crowding due to the instrument's large point-spread function (PSF, FWHM $\simeq 1$ !'6), such that simple aperture photometry might result in inaccurate fluxes due to contamination from nearby sources. To address this issue, we used a deblending technique (Eyles et al. 2005; Stark et al. 2007; Overzier et al. 2009) whereby contaminating neighbors are subtracted, by performing GALFIT (Peng et al. 2010) fits to the objects of interest and all their close neighbors simultaneously in a fitting window of $\sim 10^{\prime \prime} \times 10^{\prime \prime}$ around the source of interest. The IRAC PSFs were created from the isolated stars in the same image. For each source, we chose the PSF from nearby stars with a balance between brightness and isolation.

Positions and radial profiles of neighboring sources in the region around a candidate were derived from the HST F160Wband mosaic and fixed (except for those of the bright and extended galaxies) after adjusting a small offset between the HST and IRAC images. All other parameters were allowed to vary within preset ranges. The initial input magnitudes were obtained by running SExtractor on the IRAC images and partitioned by the flux ratios of sources in the HST images. If one Sérsic model did not yield a good fit to an extended source, an additional PSF or Sérsic component was added in fitting. If a source is dominantly bright, we masked out several central pixels to avoid GALFIT being overwhelmed by these bright pixels. The typical $\chi$-square values of our fitting results are around 10 and can be as good as 2 when nearby sources are well subtracted. The photometric uncertainties were derived based on the fluctuations of residual images. We performed aperture photometry in the residual images with a diameter of 2 . 5 and an aperture correction of a factor of 2, and measured the flux variations as our photometric uncertainties.

\section{F435W F606W F814W Optical}

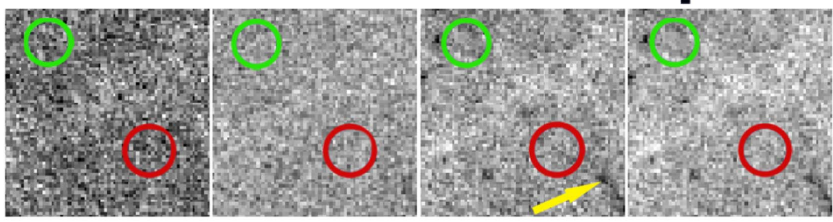

\section{F105W F125W F140W F160W}

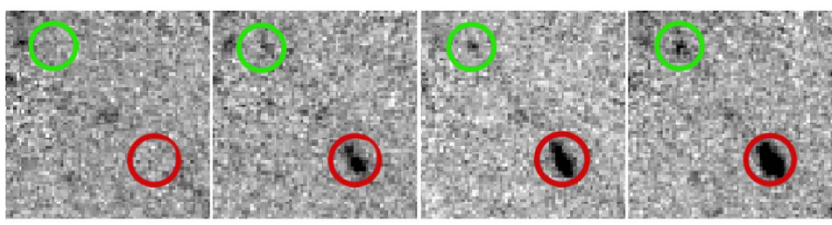

IRAC 1 IRAC 2

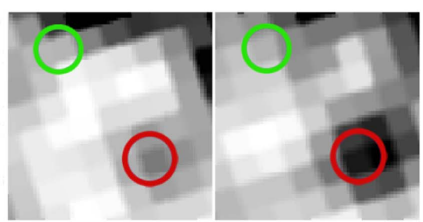

Figure 3. Cutout images of bright $z=9.44$ LBG MACS1149-JD. The object MACS1149-JD (663) is marked by red circles, and the faint object 594 by green circles. A yellow arrow in the $\mathrm{F} 814 \mathrm{~W}$ band marks a potential contaminating source. The FOV is $4 . ! 6$, north is up and east is to the left.

We then carried out our second-step selection, on the initial list of color-selected objects. Using the photometry in seven HST bands and two IRAC bands, we calculated photometric redshifts with the code BPZ (Bayesian Photometric Redshifts; Benítez 2000; Coe et al. 2006), adopting the same template library used by the CLASH collaboration (Jouvel et al. 2014). We assumed flat priors on both galaxy type and redshift in the range $z=0-12$. We required that all the candidates have a best-fit redshift solution of $z=7$ or higher, a $1 \sigma$ redshift range greater than 5 , and that the probability of $z<5$ is less than $10 \%$ of that for the high-redshift solutions. In Figures 3 and 4 we show cutout images of the $z>7$ candidates in the cluster field, and in Figure 5 those of the parallel field.

Using BPZ, we identify 25 candidates in the two fields that satisfy our color selection criteria and whose photometric redshifts (best values) place them at $z>7$. In Tables 2 and 3 we list their coordinates, photometric redshifts, photometry, and magnifications. 4 of these 25 are considered as the multiple images of a single galaxy at $z \simeq 7.1$ (see M1 and M2 in Table 2). Therefore, we consider 22 galaxy candidates in these two fields. Our IRAC analysis yields results for 17 sources for which photometry or upper limits from GALFIT were possible.

\section{Lens Models}

As part of the HFF initiative, seven strong-lensing models and one weak-lensing model were developed for MACS1149 and publicly released through the MAST archive. We used the strong-lensing models for MACS1149 as they were made to higher spatial resolutions. To estimate the systematic uncertainty in the magnification of each of our high-redshift 


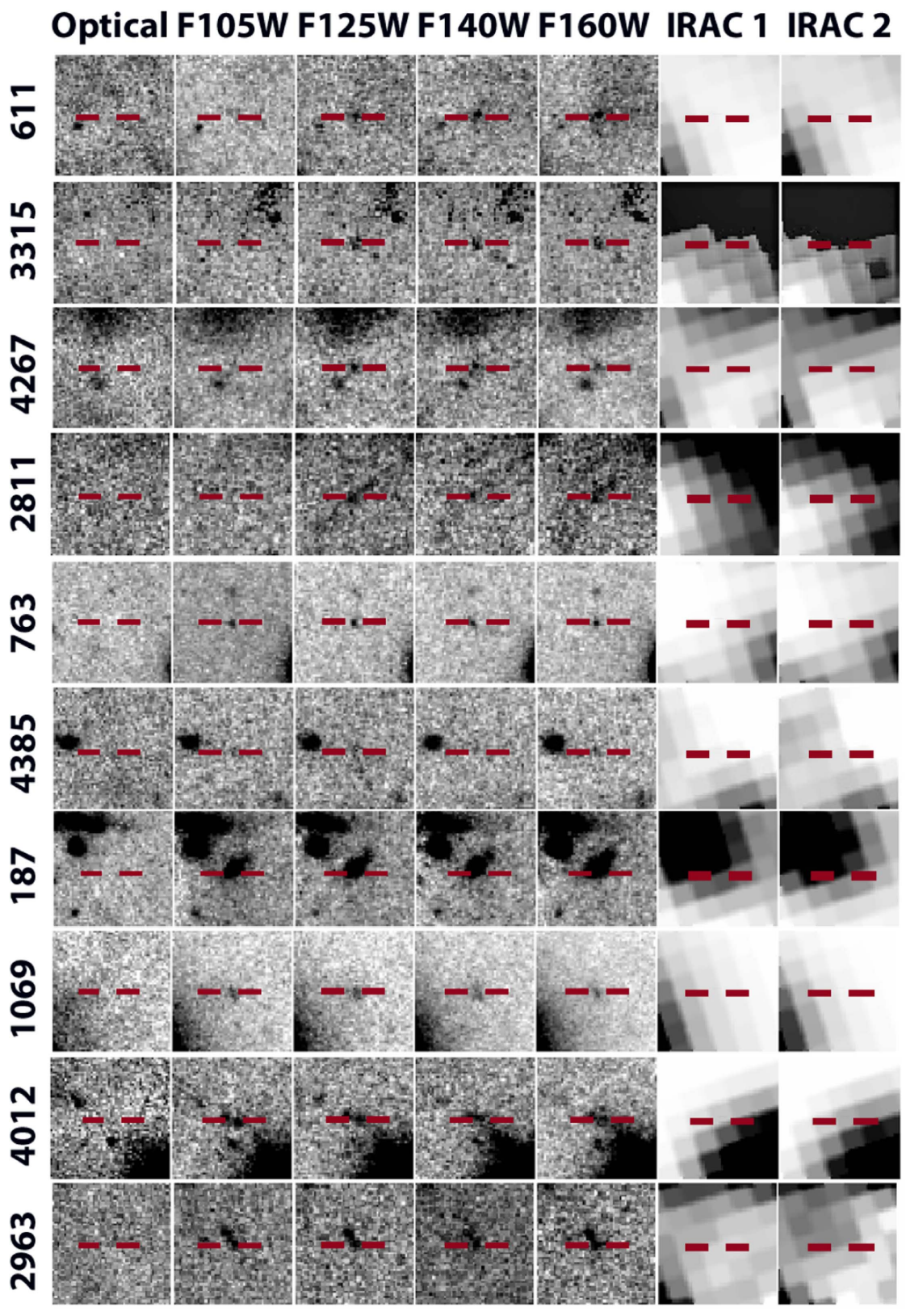

Figure 4. Cutout images of other LBG candidates of $z>7$ in MACS1149. The optical images are from the respective ACS detection images, which are the weighted sums of ACS data in the F814W, F606W, and F435W bands. Each candidate is at the image center, marked by pairs of red bars. The FOV is 3!' 3 , north is up and east is to the left.

candidates, we calculated the median magnification factor for each candidate from the seven models (Table 2) and its corresponding range for the five middle-ranked values, namely excluding the highest and lowest magnification factors. This procedure is intended to mitigate potential extremes in the model predictions, and to properly reflect the underlying systematic uncertainties.

Figure 1 shows the composite color image of the MACS1149 field, overlaid with the critical curves from the recent ZITRIN LTM (Light-Tracing-Mass) model and identification numbers for our candidates. For more details on the ZITRIN LTM model see the HFF webpage and Zitrin et al. (2015). Note that the critical curves in this best-fit model suggest that counter images for MACS1149-JD at $z \sim 9.4$ should appear, although we do not secure such an identification in the data, and the prediction is weak given the lack of constraints in that region of the cluster. In other words, the exact position of the critical curves in this northern region is uncertain (see also Kawamata et al. 2016, K16 hereafter). We discuss this further in Section 5. Additionally, the predicted positions of some counter images are close to a member galaxy near the critical curve, making predictions in that area difficult and sensitive to small changes in the mass of this member galaxy.

The objects in the parallel field are far away from the lensing field, but still experience weak-lensing effects. Using the weaklensing model of J. Merten, we derived magnification factors for the sources in Table 3 between 0.95 and 1.2. 


\section{Optical F105W F125W F140W F160W IRAC 1 IRAC 2}

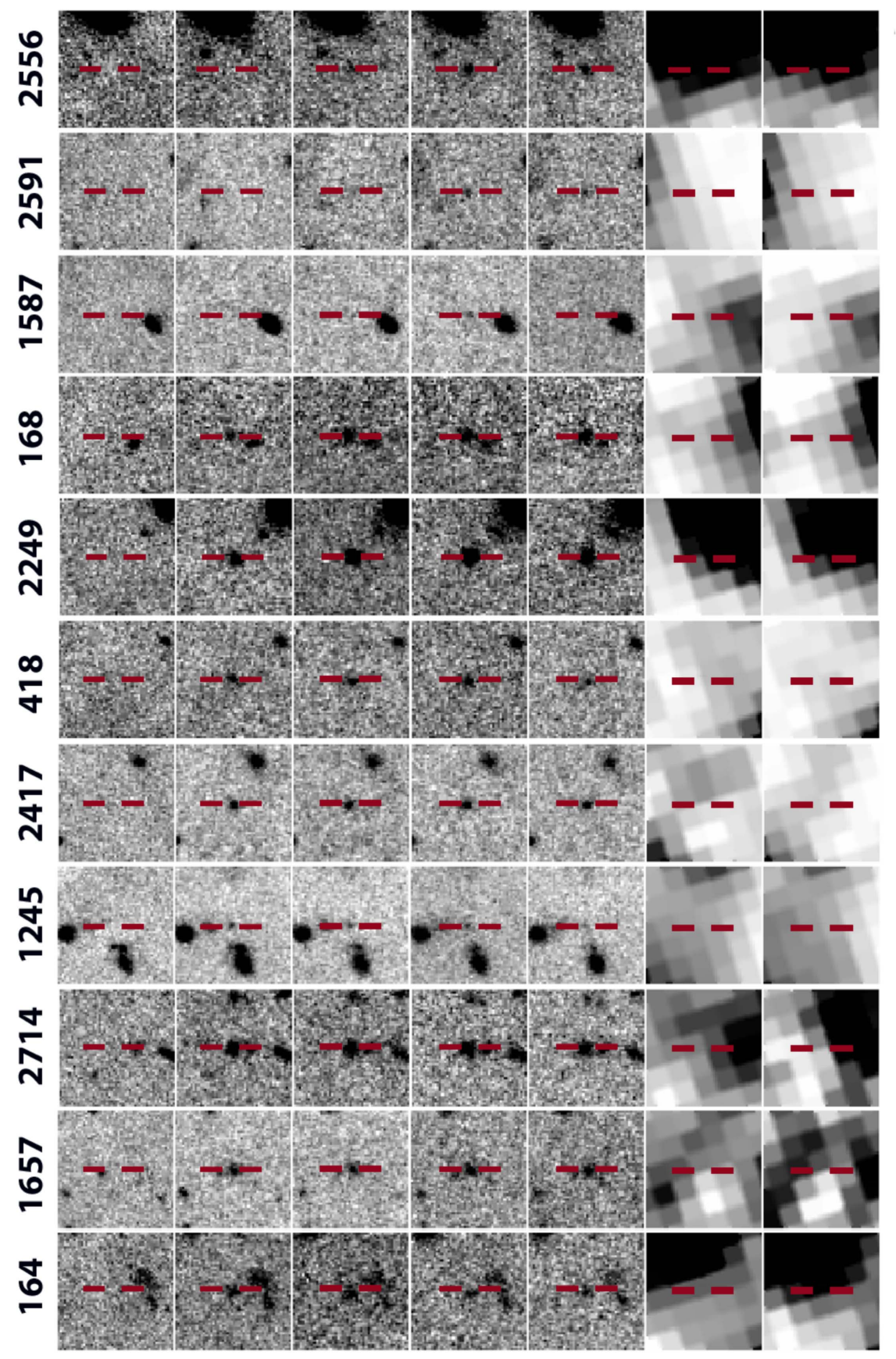

Figure 5. Cutout images of LBG candidates of $z>7$ in the MACS1149 parallel field. The symbols are the same as Figure 4.

\section{Discussion}

\subsection{MACS1149-JD}

The $z \sim 10$ dropout MACS1149-JD, detected by Z12 using shallower CLASH HST imaging and shallower IRAC data, remains robust. Now that considerably deeper HST and Spitzer data are available, we revisit the properties of this object. As shown in Table 2, it remains undetected in the F105W, F606W, and F435W bands, but weakly detected in the F814W band. We inspected the F814W image and found that the residual flux is not centered at the source. There appears to be a region of extended emission of approximately 1 arcsec in size toward the west direction. This extended emission appears to be associated with a source approximately one arcsecond south-west away (marked with a yellow arrow in Figure 3). It is so weak that it can be seen only in the F814W and F105W bands. Even with this possible contamination, the color decrement between $\mathrm{F} 140 \mathrm{~W}$ and $\mathrm{F} 814 \mathrm{~W}$ is at least $3.5 \mathrm{mag}$, further reducing the possibility of a low-redshift nature. We ran BPZ with the new data, and found a slightly updated redshift of $z=9.44 \pm 0.12$, in good agreement with our previous estimate of $z=9.6 \pm 0.2$. The probability of being a low-redshift object is less than $10^{-10}$. MACS1149-JD is therefore photometrically reconfirmed within an accurate redshift range. The source is well detected in both the IRAC1 and 2 bands, allowing an improved estimate of its age and star formation rate (SFR). 
Table 3

Candidates at $z>7$ in the MACS1149 Parallel Field

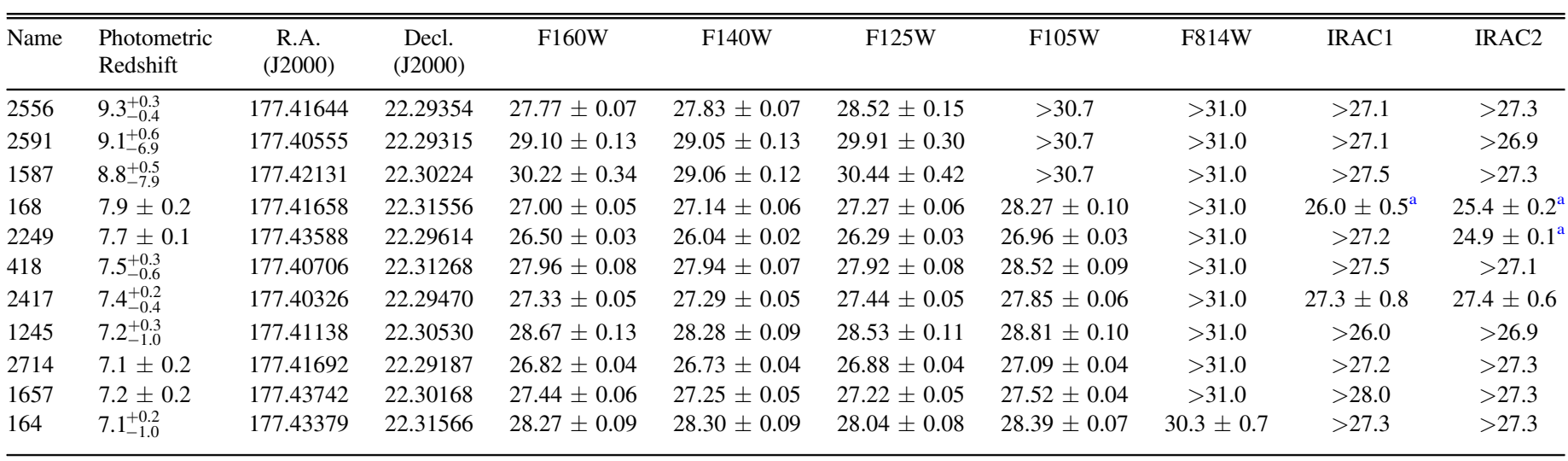

Note.

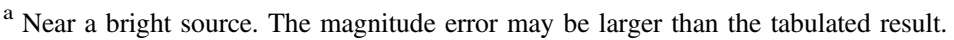

The seven predicted magnification factors of MACS1149-JD are: 9.18 for CATS (Richard et al. 2014); 12.73 for Lenstool (Johnson et al. 2014); 8.48 for Zitrin LTM; 11.56 for Zitrin LTM-Gauss (Zitrin et al. 2013); 17.0 for GLAFIC (Ishigaki et al. 2015); 4.8 for GRALE (Grillo et al. 2015); and 14.9 for Bradač \& Hoag (Bradač et al. 2009). The new models yield estimates of its magnification factor as $11.5_{-3.1}^{+3.3}$.

MACS1149-JD was not detected in IRAC1 in Z12 because of the shallow IRAC data that were available. With deeper IRAC data, Bradač et al. (2014) and Huang et al. (2016) found the source's IRAC1 magnitude as $25.7 \pm 0.5$, close to our measurement. The latest IRAC data enable an accurate estimate of the source's intrinsic properties in the optical bands. The flux in IRAC2 is brighter than IRAC1 by $\approx 0.9$ mag, likely the result of a Balmer break.

In order to derive the galaxy's physical properties, we used the modeling code iSEDfit (Moustakas et al. 2013) to fit the source's spectral energy distribution (SED). With a Monte Carlo technique, we generated 20,000 model SEDs with a broad range of star formation histories, ages, stellar metallicities, dust contents, and nebular emission-line strengths. Our models in particular included nebular emission lines whose luminosity is tied self-consistently to the number of hydrogenionizing photons. For more details of isEDfit see Z12, Zheng et al. (2014) and Infante et al. (2015).

Figure 6 displays the results for MACS1149-JD, with a fixed $\mathrm{BPZ}$ value of $z=9.44$. The derived SFR is $1.5 \pm 0.2$ $(10 / \mu) M_{\odot}$ per year, with stellar mass of $6 \times 10^{8}-$ $10^{9}(10 / \mu) M_{\odot}$, where $\mu$ is the magnification factor. The most interesting parameter is probably the galaxy age of $185 \pm 60 \mathrm{Myr}$, implying a formation redshift of $z \simeq 13.2 \pm 1.7$. The significant decrement between IRAC1 and 2 are largely explained by a Balmer decrement. The color between IRAC1 and F160W represents the UV continuum slope, which appears to be flat in $F_{\nu}$ and shows no sign for dust extinction.

Object 594 in the vicinity is consistent with $z \simeq 9$. If physically related, they are separated by about one arcsecond (4.4 kpc in the source plane). While it is possible that these are counter images of the same object (see K16) due to lensing, it is unlikely: If the critical curve at $z \simeq 9.5$ is located between these two objects, they should be of roughly similar
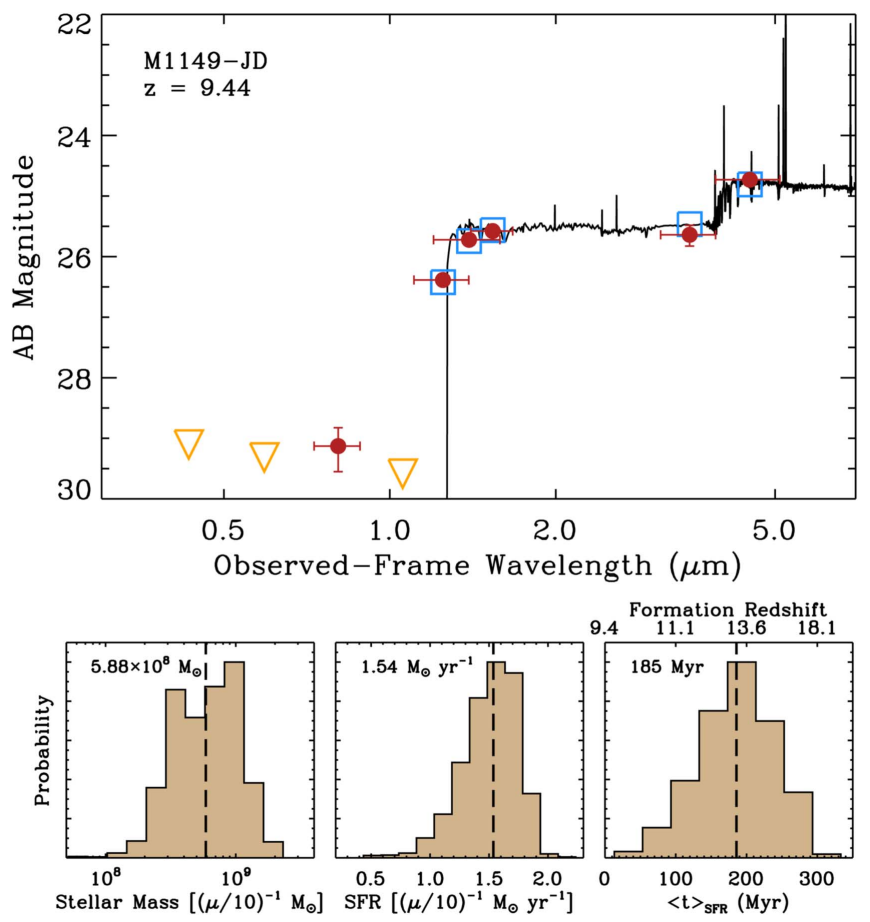

Figure 6. SED of MACS1149-JD. In the top panel, the filled red points show the observed photometry, while the open brown triangles indicate $2 \sigma$ upper limits. The black spectrum shows the best-fit (maximum likelihood) SED based on our Bayesian SED modeling using iSEDfit. The blue squares show the photometry of the best-fit model convolved with the ACS, WFC3, and IRAC filter response curves. In the bottom panel, the probability of three fitted parameters are displayed.

brightness and, in any case, the surface brightness must be the same. Pairs of multiple images should also have been found for nearby background galaxies at lower redshifts. The LTM model predicts counterpart images, but none were found. Some other lensing models, including the previous versions of ZITRIN LTM model, place MACS1149-JD outside the $z=9$ critical curve so that no multiple images are predicted. It should be noted that there is lack of multiple image constraints in the northern part of the critical curves and thus its extent in this region is poorly constrained. Given the depth of the HFF data, it becomes unlikely that MACS1149-JD is multiply imaged. 


\subsection{Multiple Images}

To help corroborate the high-redshift nature of our candidates in the cluster field, we searched for potential counter images near the locations predicted by the ZITRIN LTM gravitational lensing model as well as that of Diego et al. (2016). Objects M1 and M2 (see Figure 1) display similar redshifts and are at the positions accurately predicted by the lensing models. Both images are binary, as shown in the lower two rows of Figure 4. Because of the high magnification, the intrinsic separation between the components is about 0 !' 2 , or $0.8 \mathrm{kpc}$. Each of these components is unresolved even under significant magnification. We carried out image deconvolution of M2 using the Lucy-Richardson algorithm in 20 iterations, as the sources are in a region clear of contaminations. The reference PSF image was derived from a field star. The resultant image sizes are $\lesssim 1.5$ pixel or 0 " 1 in half-light radius. From a magnification factor of 7 , we derived an intrinsic magnitude of 29.8 and $\log r_{h l} \simeq-1.4$. The result, together with that for MACS1149-JD (Z12), is consistent with the expectation from a size-luminosity relation $r_{h l} \propto L^{-0.5}$ (Figure 9, Bouwens et al. 2016). The lensing models also predict a third image, but its position is uncertain, and no counterpart source is found with confidence. For faint counter images predicted near bright cluster galaxies (BCG), we used BCG-subtracted images to carry out our search. Several potential candidate dropouts are seen nearby, albeit none of these pass our selection criteria.

Our lensing models predict that object 3315 is highly magnified and has at least two counterpart images. One of the potential candidates is at R.A. $=177.39724$ and decl. $=22.39125$. While it displays a color similar to that at $z \sim 8$, its faint magnitude of 29 does not yield a high BPZ value. Another image is predicted in the northeast part of the field (near object 1069) but likely undetected because of the low magnification.

\subsection{Comparison with Other Work}

Among the 14 sources listed in Table 2, 11 have been reported in K16. Object 4385 is the faintest candidate, therefore it may fall below their detection threshold. For candidates 4267 and 2811, the photometry of the public HFF images confirmed independently the BPZ values $z \sim 8.2$ for these two candidates.

The pairs M1 and M2 were also identified in K16 as individual components at $z \simeq 6$. Since they are undetected or in the F814W band, their redshifts should be similar and close to 7. Object YJ3 in K16 was not confirmed in our analysis because a weak detection in the F814W band places the source at redshift between 6 and 7. It is close to a red diffuse source and may be subject to contamination.

\section{Conclusion}

We searched for high-redshift dropout candidates in the HFF cluster MACS1149. We found 22 LBG candidates at $z>7$ in the cluster field and the parallel field, reaching an intrinsic UV magnitude of $\approx-15.5$. Two of the candidates are image pairs of a single galaxy. Three of them are detected in the Spitzer/ IRAC images. We also used the new, deep data to revise the fit to the previously reported $z \sim 9.6$ candidate MACS1149-JD (Z12). The deeper data support the high-redshift solution for MACS1149-JD (it is not detected in the deeper optical images), and the updated fitting suggests a photometric redshift of $z=9.44 \pm 0.12$, stellar mass of $(7 \pm 2) \times 10^{8} M_{\odot}$, star formation rate of $\simeq 1.5 M_{\odot}$ per year, and a formation redshift of $z=13.2_{-1.6}^{+1.9}$ (age of $\approx 300 \mathrm{Myr}$ ). Our results show once more the usefulness of using gravitational lenses for accessing the faint, early universe. Aided by gravitational lensing, we have found galaxies as faint as $0.01 L^{*}$, a remarkable depth for observing galaxies at the heart of the reionization era, in particular, in the advent of the James Webb Space Telescope.

The work presented in this paper is based on observations made with the NASA/ESA Hubble Space Telescope, obtained from the Data Archive at the Space Telescope Science Institute, which is operated by the Association of Universities for Research in Astronomy, Inc., under NASA contract NAS 5-26555. It is also based on data obtained with the Spitzer Space Telescope, which is operated by the Jet Propulsion Laboratory, California Institute of Technology under a contract with NASA. This work utilizes gravitational lensing models produced by the teams led by Bradač, Ishigaki, Kneib \& Natarajan, Sharon, Williams, Merten \& Zitrin, respectively. Support for A.Z. is provided by NASA through Hubble Fellowship grant HST-HF51334.01-A awarded by STScI. N.L. acknowledges support from a European Research Council Advanced Grant FP7/ 669253. L.I., N.L., F.E.B. and P.T.I. are in part supported by CONICYT-Chile grants Basal-CATA PFB-06/2007, 3140542 and Conicyt-PIA-ACT 1417. F.E.B. also thanks CONICYTChile grant FONDECYT Regular 1141218 and the Ministry of Economy, Development, and Tourism's Millennium Science Initiative through grant IC120009, awarded to The Millennium Institute of Astrophysics, MAS. J.M.D. acknowledges support of the consolider projects CSD2010-00064, AYA2012-39475-C0201 and AYA2015-64508-P (MINECO/FEDER, UE). A.M. acknowledges the financial support of the Brazilian funding agency FAPESP (Post-doc fellowship 2014/11806-9). X.X.H. and J.X.W. acknowledge support from the National Science Foundation of China (grants 11233002,11421303) and the CAS Frontier Science Key Research Program (QYZDJSSWSLH006).

\section{References}

Atek, H., Richard, J., Jauzac, M., et al. 2015, ApJ, 814, 69 Atek, H., Richard, J., Kneib, J.-P., et al. 2014, ApJ, 786, 60 Benítez, N. 2000, ApJ, 536, 571

Bertin, E., \& Arnouts, S. 1996, A\&AS, 117, 393

Bouwens, R. J., Illingworth, G. D., Franx, M., \& Ford, H. 2008, ApJ, 686,230

Bouwens, R. J., Illingworth, G. D., Oesch, P. A., et al. 2010, ApJ, 708, 69 Bouwens, R. J., Illingworth, G. D., Oesch, P. A., et al. 2012, ApJL, 752, L5 Bouwens, R. J., Illingworth, G. D., Oesch, P. A., et al. 2015, ApJ, 803, 34 Bouwens, R. J., Illingworth, G. D., Oesch, P. A., et al. 2016, arXiv:1608.00966 Bradač, M., Russell, R., Stefano, C., et al. 2014, ApJ, 785, 108 Bradač, M., Treu, T., Applegate, D., et al. 2009, ApJ, 706, 1201 Coe, D., Benítez, N., Sánchez, S. F., et al. 2006, ApJS, 132, 926 Diego, J. M., Broadhurst, T., Chen, C., et al. 2016, MNRAS, 456, 356 Ebeling, H., Barrett, E., Donovan, D., et al. 2007, ApJL, 661, L33 Eyles, L., Bunker, A., Stanway, E., et al. 2005, MNRAS, 364, 443 Grillo, C., Suyu, S. H., Rosati, P., et al. 2015, ApJ, 800, 38 Huang, K.-H., Bradač, M., Lemaux, B. C., et al. 2016, ApJ, 817, 11 Infante, L., Zheng, W., Laporte, N., et al. 2015, ApJ, 815, 18 Ishigaki, M., Kawamata, R., Ouchi, M., et al. 2015, ApJ, 799, 12 Johnson, T. L., Sharon, K., Bayliss, M. B., et al. 2014, ApJ, 797, 48 Jouvel, S., Høst, O., Lehav, O., et al. 2014, A\&A, 562, A86 Kawamata, R., Oguri, M., Ishigaki, M., Shimasaku, K., \& Ouchi, M. 2016, ApJ, 819, 114 (K16)

Kelly, P. L., Rodney, S. A., Treu, T., et al. 2015, Sci, 347, 1123

Kelly, P. L., Rodney, S. A., Treu, T., et al. 2016, ApJL, 819, L8

Koekemoer, A. M., Mack, J., Lotz, J. M., et al. 2016, BAAS, 228, 315.02 Laporte, N., Infante, L., Troncoso Iribarren, P., et al. 2016, ApJ, 820, 98 Livermore, R. C., Feikelstein, S. L., \& Lotz, J. M. 2016, arXiv:1604:06799 
Loeb, A., \& Barkana, R. 2001, ARAA, 39, 19

Lotz, J. M., Koekemoer, A., Coe, D., et al. 2016, arXiv:1605.06567

Makovoz, D., \& Khan, I. 2005, in ASP Conf. Ser. 132, Astronomical Data Analysis Software and Systems VI, ed. P. L. Shopbell, M. C. Britton, \& R. Ebert (San Francisco, CA: ASP), 81

McLeod, D. J., McLure, R. J., Dunlop, J. S., et al. 2015, MNRAS, 450, 3032

Moustakas, J., Coil, A., Aird, J., et al. 2013, ApJ, 767, 50

Overzier, R. A., Shu, X., Zheng, W., et al. 2009, ApJ, 704, 548

Peng, C. Y., Ho, L. C., Impey, C. D., \& Rix, H.-W. 2010, AJ, 139, 2097

Postman, M., Coe, D., Benítez, N., et al. 2012, ApJS, 199, 25

Richard, J., Jauzac, M., Limousin, M., et al. 2014, MNRAS, 444, 268

Robertson, B. E., Ellis, R. S., Furlanetto, S. R., \& Dunlop, J. S. 2015, ApJL, 902, L19

Rodney, S. A., Strolger, L.-G., Kelly, P. L., et al. 2016, ApJ, 820, 50

Smith, G. P., Ebeling, H., Limousin, M., et al. 2009, ApJL, 707, L163
Stark, D. P., Bunker, A. J., Ellis, R. S., Eyles, L. P., \& Lacy, M. 2007, ApJ, 659,84

Treu, T., Brammer, G., Diego, J. M., et al. 2016, ApJ, 817, 60

Wang, W.-H., Barger, A. J., \& Cowie, L. L. 2012, ApJ, 744, 155

Zheng, W., Bradley, L. D., Saraff, A., et al. 2012a, in Seventh Conf. on Astronomical Data Analysis, APLUS: A Data Reduction Pipeline for HST/ ACS and WFC3 Images, 17

Zheng, W., Postman, M., Zitrin, A., et al. 2012b, Natur, 489, 406 (Z12)

Zheng, W., Shu, X., Moustakas, J., et al. 2014, ApJ, 795, 126

Zitrin, A., \& Broadhurst, T. 2009, ApJ, 703, 132

Zitrin, A., Broadhurst, T., Barkana, R., Rephaeli, Y., \& Benítez, N. 2011, MNRAS, 410, 1939

Zitrin, A., Fabris, A., Merten, J., et al. 2015, ApJ, 801, 44

Zitrin, A., Meneghetti, M., Umetsu, K., et al. 2013, ApJ, 762, 30

Zitrin, A., Zheng, W., Broadhurst, T., et al. 2014, ApJL, 793, L12 